\title{
Cholesterol metabolite unmasked as a key player in breast cancer
}

Mechanisms underpinning the role of elevated cholesterol in breast cancer risk are poorly understood. However, researchers from Duke University now reveal that 27-hydroxycholesterol (27HC), a primary metabolite of cholesterol, can both speed up the growth of breast cancer and promote its spread to the lungs.

Building on their previous work, which identified 27HC as an estrogen-receptor (ER) agonist, the researchers showed that the effects of cholesterol on the growth of ER-positive tumours requires its conversion to $27 \mathrm{HC}$. Blocking the enzyme that catalyses this reaction (CYP27A1; also known as mitochondrial sterol 26-hydroxylase) delayed tumour onset in cancer-prone mice, even those fed a high-cholesterol diet. Notably, the effects of $27 \mathrm{HC}$ on metastasis were independent of those on growth and did not involve the ER.

"The finding that elevated cholesterol (and 27HC) dramatically shortens the time needed for animals to get cancer really caught our attention," states lead investigator Donald McDonnell. "What is most exciting was the observation that lowering cholesterol alone in animals was sufficient to reduce breast cancer risk." Consequently, taking steps to reduce cholesterol levels with lipid-lowering drugs might be a viable option for primary prevention, as well as treatment of women with breast cancer.

"We have recently received funding to define the mechanisms by which 27HC impacts metastasis and to evaluate the utility of statins and inhibitors of CYP27A1 as therapeutic modalities," McDonnell concludes.

Vicky Heath

Original article Nelson, E. R. et al.

27-Hydroxycholesterol links hypercholesterolemia and breast cancer pathophysiology. Science doi:10.1126/ science. 1241908 